\title{
Models of Neutrino Mass with a Low Cutoff Scale
}

\author{
Hooman Davoudiasl, ${ }^{1,2}$ Ryuichiro Kitano, ${ }^{2}$ Graham D. Kribs, ${ }^{2,3}$ and Hitoshi Murayama ${ }^{4,5,2}$ \\ ${ }^{1}$ Department of Physics, University of Wisconsin, Madison, WI 53706 \\ ${ }^{2}$ School of Natural Sciences, Institute for Advanced Study, Princeton, NJ 08540 \\ ${ }^{3}$ Department of Physics, University of Oregon, Eugene, OR 97403* \\ ${ }^{4}$ Department of Physics, University of California, Berkeley, CA 94720 \\ ${ }^{5}$ Theoretical Physics Group, Lawrence Berkeley National Laboratory, Berkeley, CA 94720
}

\begin{abstract}
In theories with a low quantum gravity scale, global symmetries are expected to be violated, inducing excessive proton decay or large Majorana neutrino masses. The simplest cure is to impose discrete gauge symmetries, which in turn make neutrinos massless. We construct models that employ these gauge symmetries while naturally generating small neutrino masses. Majorana (Dirac) neutrino masses are generated through the breaking of a discrete (continuous) gauge symmetry at low energies, e.g., $2 \mathrm{keV}-1 \mathrm{GeV}$. The Majorana case predicts $\Delta N_{\nu} \simeq 1$ at BBN, neutrinoless double beta decay with scalar emission, and modifications to the CMB anisotropies from domain walls in the universe as well as providing a possible Dark Energy candidate. For the Dirac case, despite the presence of a new light gauge boson, all laboratory, astrophysical, and cosmological constraints can be avoided.
\end{abstract}

\section{INTRODUCTION}

The hierarchy between the electroweak scale and the Planck scale is one of the most puzzling aspects of the Standard Model (SM). On the one hand, allowing the cutoff scale of the SM to be the Planck scale allows us to naturally understand both the stability of the proton and the smallness of the neutrino masses through the seesaw mechanism. On the other hand, an enormous finetuning is required to prevent the Higgs mass from being destabilized by radiative corrections proportional to the cutoff scale. Our options appear to be to either live with fine-tuning (for example [1]) or find a dynamical solution that may well affect the successes of the Standard Model with a high cutoff scale.

The most dramatic solution to the hierarchy problem is to lower the cutoff scale of the SM close to the weak scale. In scenarios with large or warped extra dimensions [2, 3], the cutoff scale of the SM is the fundamental scale of quantum gravity. All global symmetries are expected to be violated by quantum gravity effects, in particular baryon and lepton number are violated leading to extremely rapid proton decay and way-too-large Majorana neutrino masses. There are intrinsically extra dimensional solutions to these problems 迎, 5], for example, by separating fermions within a fat brane. The solution to the too-large Majorana neutrino mass problem, however, requires more structure beyond fermion geography. ${ }^{1}$

\footnotetext{
${ }^{*}$ On leave of absence.

${ }^{1}$ Consider, e.g., the interesting proposal of 4 , in which $\mathrm{U}(1)_{B-L}$ is gauged in the bulk. The needed geography is to separate lefthanded neutrinos from right-handed neutrinos from $\mathrm{U}(1)_{B-L}$ breaking fields in the extra dimensional space. It also requires additional scalar fields with suitable profiles to stabilize the positions of the fermions. Furthermore, the smallest cutoff scale must be parametrically larger than the inverse distance separating left-handed and right-handed leptons to ensure the wave-
}

In this paper we consider simple, four-dimensional solutions to the problems of baryon and lepton number violation. Our proposals are applicable to models of large extra dimensions, as well as any other model that lowers the cutoff scale of the SM, violating the global symmetries. We propose to avoid those problems by imposing new gauge symmetries in the effective theory that forbid the dangerous operators. Baryon number and lepton number violation are eliminated (up to $\Delta B=3$ and $\Delta L=3$ ) by imposing a gauged $Z_{9}$ baryon number and a gauged $Z_{3}$ lepton number. Similar gauged discrete symmetries were employed in [6, 7 .

Gauging discrete baryon number eliminates the proton decay problem. Gauging discrete lepton number causes the neutrinos of the SM to be massless. Since the cutoff scale is low, we lose the usual seesaw mechanism as an explanation for the smallness of neutrinos masses. Here we will consider two models to generate neutrino masses of the experimentally observed size. The first is to break $Z_{3}$ lepton number by a small amount, generating small Majorana neutrino masses. The second is to introduce right-handed neutrinos and a new U(1) gauge symmetry that is spontaneously broken at a scale deep in the infrared, generating small Dirac neutrino masses. We subject our models to severe experimental, astrophysical, and cosmological constraints and demarcate the regions of parameter space that survive. Remarkably, despite the presence of a new scalars and/or a gauge boson with mass well below the electroweak scale, we find these models provide viable solutions to the problems of baryon and lepton number violation from low cutoff scale operators.

The models we present are similar to those in Refs. 88, 9], in that neutrino mass originates from a symmetry breaking at low scales, and hence a phase transition at late times. There is, however, an important differ-

function overlaps are sufficiently small. 
ence. While they have focused on nearly massless pseudoNambu-Goldstone bosons due to spontaneously broken global symmetries, we insist on all symmetries to be gauged and we do not have any nearly massless bosons. Therefore, in general, the phenomenological constraints are correspondingly quite different. We note that models with a low cutoff scale based on discrete symmetries, but without dynamics in the infrared regime we consider, have been studied in Ref. [7].

The paper is organized as follows. In the next section, we introduce flavor-blind discrete gauge symmetries that ensure the absence of too-rapid proton decay and Majorana neutrino masses. In section III, we break the discrete gauge symmetry spontaneously and induce the Majorana neutrino masses at the required size. We show that the constraints from the Big-Bang Nucleosynthesis (BBN) as well as the neutrinoless double beta decay with an additional scalar emission are marginal. The network of domain walls is an inevitable prediction, which naturally satisfies the constraint from cosmic microwave anisotropy and can be a candidate for Dark Energy. We discuss the Dirac case in section IV. We first show that an additional discrete gauge symmetry can suppress the Dirac neutrino mass, but the predicted domain wall network requires a high cutoff scale. We then focus on a new continuous gauge symmetry, which necessarily predicts a new very light gauge boson, and show that all laboratory, astrophysical, and cosmological constraints can be satisfied. Finally we comment on the hierarchy between the neutrino mass scale and the fundamental scale in section V, and conclude in section $\mathrm{VI}$.

\section{PROTECTING MATTER}

The most pressing problems of lowering the cutoff scale in the SM are fast proton decay and too large Majorana neutrino masses. We propose a set of two discrete symmetries; the SM quarks transform under one symmetry, and leptons transform under the other. Here, we have assumed that the chiral fermion content is the same as in the minimal SM. The discrete symmetries are assumed to be flavor-blind (although we comment on a non-flavorblind variation that suppresses $\mu \rightarrow e \gamma$ in Appendix A. . To ensure that the gauged discrete symmetries are not badly broken at the electroweak scale, the Higgs is taken to be neutral. Allowing ordinary Yukawa couplings enforces the conditions $Z_{A}^{q}(Q)=-Z_{A}^{q}\left(u^{c}\right)=-Z_{A}^{q}\left(d^{c}\right)$ and $Z_{B}^{\ell}(L)=-Z_{B}^{\ell}\left(e^{c}\right)$. This leaves us with

$$
\begin{array}{c|ccccc} 
& Q & u^{c} & d^{c} & L & e^{c} \\
\hline Z_{A}^{q} & a & -a & -a & 0 & 0 \\
Z_{B}^{\ell} & 0 & 0 & 0 & b & -b
\end{array}
$$

The $Z_{A}^{q}-S U(3)$-SU(3) and $Z_{A}^{q}$-grav-grav anomalies are canceled for any $a, A$. The $Z_{A}^{q}-S U(2)-S U(2)$ anomaly requires $9 a=0 \bmod A$. We choose $(A, a)=(9,1)$. Similarly the $Z_{B}^{\ell}$-SU(2)-SU(2) and $Z_{B}^{\ell}$-grav-grav anomalies require $3 b=0 \bmod B$, and hence $(B, b)=(3,1)$. Mixed $U(1)$-discrete anomalies can be satisfied by redefining the $U(1)$ normalization, and pure discrete anomalies can be satisfied by adding additional massive matter with suitable (fractional) discrete charges [6, 10]. Note that these discrete symmetries, up to certain charge redefinitions, have been proposed before in supersymmetric [6] and non-supersymmetric [7] contexts. In particular, our $Z_{3}^{\ell}$ is equivalent to $L_{3}$ of [6]. Our $Z_{9}^{q}$ is equivalent to $R_{3}^{-3} L_{3}^{-3} Y$ of [6] where $R_{3}^{-3}, L_{3}^{-3}$ is understood to mean $R_{3}, L_{3}$ promoted to $R_{9}, L_{9}$ with all fields' discrete charge multiplied by -3 , and $Y$ refers to the $Z_{9}$ subgroup of $U(1)_{Y}$.

The lowest dimension operators leading to baryon and lepton number violation are schematically

$$
\frac{Q^{9} L^{3}}{\Lambda^{14}} \quad+\text { others... }
$$

The most conservative assumption is to require this $\Delta B=3$ process leads to a multi-nucleon lifetime that is longer than the proton lifetime,

$$
\tau=\frac{\Lambda^{28}}{m_{\text {nuc }}^{29}}>10^{33} \mathrm{yrs}
$$

and thus $\Lambda \gtrsim 200 m_{n u c}$. This constraint is easily satisfied for the cutoff scales we consider in this paper. Similarly Majorana neutrino mass is completely forbidden so long as $Z_{3}^{\ell}$ is exact.

\section{III. $Z_{3}$ BREAKING AND MAJORANA NEUTRINOS}

With exact $Z_{3}^{\ell}$ lepton number symmetry, Majorana neutrino masses arising from cutoff scale operators are forbidden. To generate non-zero neutrino masses, of Majorana type, the $Z_{3}$ symmetry must be broken. We show that a low breaking scale is needed, of order $2-400 \mathrm{keV}$, to give a consistent framework. Discrete symmetry breaking leads to domain walls, that can induce a too large anisotropy in the CMB. However, a frustrated network of domain walls may avoid this constraint and even provide a candidate for dark energy.

To generate neutrino masses that are purely Majorana, $Z_{3}^{\ell}$ is broken by a complex scalar field $\chi$ with $Z_{3}^{\ell}$ charge that acquires a vev. Without loss of generality we can choose the $Z_{3}^{\ell}$ charge of $\chi$ to be equal to the leptons. This allows us to write the dimension- 6 operator

$$
c \frac{\chi L H L H}{\Lambda^{2}}
$$

with a coefficient that we assume takes on a natural value, $c \sim 1$. The other nontrivial choice of discrete charge for $\chi$, namely $Z_{3}^{\ell}(\chi)=2$, leads to the same dimension- 6 operator upon simply replacing $\chi \rightarrow \chi^{*}$ in the above. The effective Yukawa coupling is

$$
g \nu \nu \chi, \quad g=c \frac{v^{2}}{\Lambda^{2}}
$$


giving a neutrino mass

$$
m_{\nu}=g\langle\chi\rangle=(0.06 \mathrm{eV}) c\left(\frac{\langle\chi\rangle}{2 \mathrm{keV}}\right)\left(\frac{30 \mathrm{TeV}}{\Lambda}\right)^{2} .
$$

An important constraint on the Yukawa coupling $g$ arises from $0 \nu \beta \beta \chi$ process where the $\chi$ is emitted in the $0 \nu \beta \beta$ transition [11]. Assuming a natural potential where $m_{\chi} \sim\langle\chi\rangle$, a physical $\chi$ is easily emitted in the nuclear transition $0 \nu \beta \beta \chi$ with no kinematic suppression. Experimental constraints on this process have determined that the effective coupling of $\chi \nu \nu$ must be smaller than $3 \times 10^{-5} 12$. This implies $\Lambda>30 \mathrm{TeV}$, taking $c=1$, independent of any other assumptions. The smallest neutrino mass that can explain the atmospheric neutrino oscillation data is approximately $\sqrt{\Delta m_{\mathrm{atm}}^{2}} \simeq 0.06 \mathrm{eV}$, which implies $\langle\chi\rangle>2 \mathrm{keV}$ from Eq. (5).

Spontaneous breaking of a discrete symmetry can lead to a domain wall problem. A network of domain walls typically "scales," i.e., the walls stretch and simplify their configuration and there is basically always only one (or a few) of the walls within the horizon. The energy density of the walls is approximately $\sigma / L$, where $\sigma \sim\langle\chi\rangle^{3}$ is the wall tension and $L \sim H^{-1}$ is the typical inter-wall distance. This implies the energy density of the walls is

$$
\Omega_{\mathrm{wall}} \sim \frac{\sigma H}{M_{P l}^{2} H^{2}} \sim 10^{-12}\left(\frac{\langle\chi\rangle}{2 \mathrm{keV}}\right)^{3}
$$

which implies the upper bound $\langle\chi\rangle<25 \mathrm{MeV}$ such that the walls do not overclose the universe. However, there is a more stringent constraint from the cosmic microwave background $(\mathrm{CMB})$ photons being affected by the walls themselves. The simple estimate is that $\Delta T / T \sim G_{N} \sigma L$ [13]. The observational bound $\Delta T / T \lesssim 10^{-5}$ requires $\sigma \sim\langle\chi\rangle^{3} \lesssim(400 \mathrm{keV})^{3}$, which gives the upper bound $\Lambda \lesssim 400 \mathrm{TeV}$. Smaller breaking scales may still leave an interesting imprint on the power spectrum or galaxy distribution functions. Unfortunately we are not aware of a detailed study of the imprint of a domain wall network due to a late time phase transition, and we cannot make any further quantitative statements.

It is possible that the domain wall network is frustrated, namely that the network is so complicated that it cannot simplify its configuration easily and the configuration expands without simplifying. It may happen if $Z_{3}$ is embedded into a larger non-Abelian discrete group. In this case, the domain wall network can behave as dark energy with $w=-2 / 3$. Even though the current data do not prefer this possibility, analyses without priors actually allow it 14], and it will surely be interesting to investigate this possibility in forthcoming supernovae studies such as SNAP.

There is a prediction on the effective number of neutrinos at the time of BBN. The $\chi$ particle can be generated in early universe through processes such as $\nu \nu \rightarrow \chi$, $\nu \nu \rightarrow \chi \chi$, and $\nu \nu \rightarrow \chi \chi \chi$. Although the $\nu \nu \rightarrow \chi \chi \chi$ process has the largest interaction rate for reasonable range of $\Lambda$, we can give a parameter independent conclusion with the former two processes. The interaction rate of those processes at temperature $T$ is roughly given by

$$
\Gamma \sim \frac{m_{\nu}^{2}}{16 \pi T}
$$

Here, we have used the natural assumption that $m \sim$ $m_{\chi} \sim\langle\chi\rangle$, with $m$ being the coefficient of the threepoint coupling term $m \chi^{3}$. Since the interaction rate $\Gamma$ at $T=T_{B B N} \sim 1 \mathrm{MeV}$ is larger than the expansion rate $H \sim T_{B B N}^{2} / M_{P l}$ for $m_{\nu} \gtrsim 10^{-4} \mathrm{eV}$, the production process is in thermal equilibrium and thus $\Delta N_{\nu}$, the effective number of neutrinos minus three, is predicted ${ }^{2}$ to be $8 / 7$. This is rather large and in apparent disagreement with the bound $N_{\nu}^{\text {eff }}<3.4$ at $95 \%$ CL found in Ref. [15]. However, additional systematic errors in the helium abundance measurements may allow such a large effective number of neutrinos, albeit the errors on the PDG central value [16] would have to be significantly increased, as discussed in 115]. Alternatively, a large chemical potential for $\nu_{e}$ allows up to $\Delta N_{\nu} \lesssim 4.1$ [17].

One may worry about the erasure of the baryon number at high temperature due to lepton number violating interactions. It is indeed important for relatively large $m(\sim\langle\chi\rangle)$ because the lepton number erasure process becomes effective before turning off the sphaleron process. When we assign lepton number -2 for $\chi$, the process which violates lepton number is $\chi \chi \rightarrow \chi \chi^{*} \chi^{*}$ through $m \chi^{3}$ term. This process enters thermal equilibrium when temperature drops down to

$$
T_{*} \sim 300 \mathrm{GeV}\left(\frac{m}{300 \mathrm{keV}}\right)^{2 / 3} .
$$

By comparing the critical temperature of the electroweak phase transition $T_{c} \sim 300 \mathrm{GeV}$, we find it is safe for $m \lesssim 300 \mathrm{keV}$.

Here, we note that the neutrinos interact among themselves by exchanging $\chi$ with a cross section $\sigma_{\nu \nu} \sim$ $g^{4} T^{2} / m_{\chi}^{4}$. At the time of decoupling, corresponding to $T \sim 1 \mathrm{eV}$, the neutrino mean free path is longer than the horizon scale, and therefore neutrinos free-stream. However, the presence of extra degrees of freedom below the scale of BBN generates a shift $\Delta l_{k}$ in the position of the $k^{t h}$ acoustic peak of the CMB. Using the large $k$ results discussed in Ref. [8], for the model discussed in this section, we get $\Delta l_{k}=-29.3$; the SM prediction for this quantity is -23.3 . Given the sensitivity of the Planck experiment, this deviation will be measurable in the future.

The $Z_{3}^{\ell}$ symmetry could be generalized to a $Z_{3 N}$, with $N$ an integer, where the leptons are assigned charge $N$

\footnotetext{
2 There are logical possibilities to avoid such large deviation. For example, small cubic and quartic couplings $\lambda$ of $\chi$ such as $m \lesssim 10^{-3}\langle\chi\rangle$ and $\lambda \lesssim 10^{-6}$ may suppress the interaction rate sufficiently.
} 
(leaving $Z_{3 N}$ non-anomalous as before) while $\chi$ is assigned a unit charge. In this case neutrino masses come from even higher dimensional operators such as

$$
\left(\frac{\chi}{\Lambda}\right)^{N} \frac{L H L H}{\Lambda} .
$$

The symmetry breaking scale in this case is estimated to be

$$
\langle\chi\rangle \sim 10^{\frac{1}{2}\left(9-\frac{19}{N}\right)} \mathrm{GeV}\left(\frac{\Lambda}{30 \mathrm{TeV}}\right)^{1+\frac{1}{N}}
$$

where the neutrino mass was fixed to be $0.1 \mathrm{eV}$. By using $\Lambda \gtrsim 30 \mathrm{TeV}$ and $\langle\chi\rangle \lesssim 400 \mathrm{keV}$ from the constraint from the domain wall formation, we find the only viable possibility is $N=1$. Hence, our choice of Eq. (3) is unique.

\section{NEW GAUGE THEORIES AND DIRAC NEUTRINOS}

In this section, we discuss how to generate small Dirac neutrino masses. Obviously we need to introduce right-handed neutrinos. The smallness of Dirac neutrino masses can be ensured by either an additional discrete or a new continuous gauge symmetry. The former case, however, has severe cosmological constraints. The latter case predicts a new very light gauge boson, yet can satisfy all laboratory, astrophysical, and cosmological constraints depending on the dimension of the operator responsible for the neutrino masses.

\section{A. Discrete Gauge Symmetry}

The unwanted large Dirac Yukawa interaction $H L \nu_{R}$ can be forbidden by introducing a new gauge symmetry. We discuss new discrete symmetries under which only the right-handed neutrino and a scalar field $H_{R}$ transform. The Dirac neutrino masses are generated after the symmetry breaking through a higher dimensional operator as in the Majorana case. We show that the constraints from $\mathrm{BBN}$ and the domain wall formation exclude almost all the range of the cutoff scale $\Lambda$ except for a high cutoff region $\Lambda \sim 10^{8} \mathrm{GeV}$.

Since the right-handed neutrinos $\nu_{R}$ are gauge singlets, it is easy to construct a model with satisfying the anomaly-free condition. For example, a model with $Z_{3}$ symmetry and the following charge assignment trivially satisfies the condition.

\begin{tabular}{c|rrr} 
& $\nu_{R}^{1}$ & $\nu_{R}^{2}$ & $\nu_{R}^{3}$ \\
\hline$Z_{3}^{R}$ & 1 & 1 & 1 \\
$Z_{3}^{\ell}$ & -1 & -1 & -1
\end{tabular}

The new $Z_{3}^{R}$ symmetry forbids Dirac Yukawa interaction terms. The neutrino masses are generated after the spontaneous $Z_{3}^{R}$ breaking by the vev of a Higgs field $H_{R}$ through the operator:

$$
\mathcal{L}^{R}=\lambda^{\alpha i} \frac{H_{R}\left(H L^{\alpha}\right) \nu_{R}^{i}}{\Lambda},
$$

where we assigned the charges of $H_{R}$ to be $Z_{3}^{R}:-1$, and $\lambda^{\alpha i}$ are $O(1)$ coefficients. As we did in the Majorana case, we can generalize the symmetry to $Z_{3 n}^{R}$ and in this case the neutrino masses are generated through the interaction terms:

$$
\mathcal{L}^{R}=\lambda^{\alpha i}\left(\frac{H_{R}}{\Lambda}\right)^{n}\left(H L^{\alpha}\right) \nu_{R}^{i} .
$$

Since this is the general form of the interaction term for any choices of the discrete symmetry group, we do not specify the gauge group hereafter and discuss the constraint for each $n$. The symmetry breaking scale is estimated by fixing the neutrino masses as follows:

$$
\left\langle H_{R}\right\rangle \sim 10^{3-\frac{12}{n}} \mathrm{GeV}\left(\frac{\Lambda}{1 \mathrm{TeV}}\right) .
$$

Now we consider the constraint on the cutoff scale $\Lambda$ from BBN. The agreement of the light element abundances with the predictions of BBN theory does not allow more than one extra neutrino species during BBN. In our model, $\nu_{R}$ are light degrees of freedom during BBN, and hence should satisfy the constraint $\Delta N_{\nu} \lesssim 1$. At the time of the QCD phase transition, any preexisting amount of $\nu_{R}$ is diluted by a factor of order 10 and hence naturally satisfies the limit if $\nu_{R}$ 's are not in the thermal bath. Instead, the more important constraint arises from the repopulation of $R$-sector particles $\nu_{R}$ and $H_{R}$ through the interaction in Eq. (12) after the QCD phase transition.

The simplest constraint arises from dimension six operators such as

$$
\frac{\left(\bar{l} \gamma^{\mu} l\right)\left(\bar{\nu}_{R} \gamma_{\mu} \nu_{R}\right)}{\Lambda^{2}}
$$

that lead to $\nu_{R}$ production. Requiring that this process is frozen out at $T \sim 200 \mathrm{MeV}$, one finds the constraint $\Lambda>5 \mathrm{TeV}$ independent of $n$.

$R$-sector particles can also be produced through the $n$ dependent operator, Eq. (12). We first consider the case $n=1$. After electroweak symmetry breaking, neutrinos have a Yukawa interaction with $H_{R}$ :

$$
\mathcal{L}_{Y}=\frac{\lambda^{\alpha i} v}{\Lambda} \nu_{L}^{\alpha} \nu_{R}^{i} H_{R}
$$

This interaction is important for the generation of $\nu_{R}$ and $H_{R}$ through the processes $\nu_{L} \bar{\nu}_{L} \rightarrow \nu_{R} \bar{\nu}_{R}$ and $\nu_{L} \bar{\nu}_{L} \rightarrow$ $H_{R} H_{R}^{*}$ via $t$-channel $H_{R}$ and $\nu_{R}$ exchange diagrams. The abundance of these $R$-sector particles is approximately given by

$$
\xi_{R} \equiv \frac{n_{R}}{T^{3}} \sim \min \left(\frac{v^{4}}{4 \pi \Lambda^{4}} \frac{M_{P}}{T}, 1\right),
$$


where $n_{R}$ is the number density of the $R$-sector particles. Even if there is no preexisting abundance of $R$-sector particles, the small amount produced by the above processes triggers more efficient production processes such as $\nu_{L} \nu_{R} \rightarrow H_{R} H_{R}^{*} H_{R}^{*}$ and $\nu_{L} H_{R} \rightarrow \bar{\nu}_{R} H_{R} H_{R}^{*}$ through the Yukawa coupling in Eq. (15) and the four-point coupling of $H_{R}$. The interaction rate is approximately given by

$$
\Gamma \sim \xi_{R} \frac{v^{2}}{(4 \pi)^{3} \Lambda^{2}} T
$$

By requiring the interaction rate in Eq. (17) to be smaller than the expansion rate of the universe at the time of BBN $(T \sim 1 \mathrm{MeV}$ ), we obtain a lower bound on the cutoff scale to be $\Lambda \gtrsim 4 \times 10^{8} \mathrm{GeV}$ with assuming $\mathcal{O}(1)$ quartic coupling constant of $H_{R}$. The bound corresponds to the discrete symmetry breaking scale to be $\left\langle H_{R}\right\rangle \gtrsim 400 \mathrm{keV}$. Since the bound from the domain wall formation is $\left\langle H_{R}\right\rangle \lesssim 400 \mathrm{keV}$, a small coefficient for the four-point $H_{R}$ coupling allows to have a viable parameter region around $\Lambda \sim 10^{8} \mathrm{GeV}$.

If we assume that $R$-sector particles are in thermal equilibrium with standard model particles above the temperature of the QCD phase transition, the number density of $R$-sector particles are only suppressed by $1 / 10$ compared to the photon number density. In this case, a more severe constraint $\Lambda \gtrsim 10^{10} \mathrm{GeV}$ is obtained.

For $n=2, R$-sector particles are most effectively produced through dimension six operators Eq. (14) and

$$
\frac{1}{\Lambda^{2}} \bar{\nu}_{L} \gamma^{\mu} \nu_{L} H_{R}^{*} \partial_{\mu} H_{R}
$$

Again, a small amount of the $R$-sector particles is enough to quickly thermalize through the processes $\nu_{L} H_{R} \rightarrow$ $\bar{\nu}_{R} H_{R}^{*}$ and $\nu_{L} \nu_{R} \rightarrow H_{R}^{*} H_{R}^{*}$ via the dimension five operator

$$
\frac{v}{\Lambda^{2}} \nu_{L} \nu_{R} H_{R}^{2}
$$

Since the interaction rate of this process is larger at higher temperature, we require that these processes are already frozen out by the time the Universe cools to the temperature of the QCD phase transition $T \sim 200 \mathrm{MeV}$. We obtain the bound $\Lambda \gtrsim 30 \mathrm{TeV}\left(\left\langle H_{R}\right\rangle \gtrsim 30 \mathrm{MeV}\right)$ and thus this model is excluded by the domain wall constraint.

The models with $n \geq 3$ are obviously also excluded by domain wall formation since the symmetry breaking scale is larger than $100 \mathrm{MeV}$ for $\Lambda \gtrsim 1 \mathrm{TeV}$.

We conclude that BBN and domain wall formation severely constrains the model. The only non-excluded region we found is around $\Lambda \sim 10^{8} \mathrm{GeV}$, leaving intact a large hierarchy between the cutoff scale and the electroweak scale.

\section{B. A New $U(1)$ Gauge Symmetry}

Since we could not find interesting Dirac neutrino models with discrete symmetries, we then consider the models with a continuous gauged symmetry which are free from the domain wall problem. We assume right-handed neutrinos $\nu_{R}^{i}, i=1, \cdots, n_{R}$ carry $U(1)_{R}$ charges while quarks and leptons do not, because the observed matter does not appear to have any gauge interactions beyond the usual $S U(3)_{C} \times S U(2)_{L} \times U(1)_{Y}$. The right-handed neutrino charges $Q=\left(q_{1}, \cdots, q_{n_{R}}\right)$ are subject to anomaly cancellation conditions,

$$
\begin{aligned}
\sum_{i} q_{i} & =0, \\
\sum_{i} q_{i}^{3} & =0 .
\end{aligned}
$$

Also, since the right-handed neutrinos should carry $Z_{3}^{\ell}$ charge -1 to form Dirac masses, we need at least three $\nu_{R}$ 's to cancel the gravitational anomaly.

There are additional requirements on the $U(1)_{R}$ charge assignments. Ordinary dimension-4 Dirac neutrino masses $L H \nu_{R}$ should be forbidden by gauge symmetry, since the purpose of the gauge symmetry is to, at least ideally, avoid requiring unnaturally small Yukawa couplings. This means at least three neutrinos must have $U(1)_{R}$ charge. $^{3}$ Given just one scalar field that breaks the $U(1)_{R}$ symmetry, the neutrino $U(1)_{R}$ charges should be equal or opposite to that of the breaking field.

One obvious choice of $U(1)_{R}$ charges for the neutrinos is

$$
\begin{array}{r|cccc} 
& \nu_{R}^{1} & \nu_{R}^{2} & \nu_{R}^{3} & \nu_{R}^{4} \\
\hline Q_{R} & +1 & -1 & +1 & -1 \\
Z_{3}^{\ell} & -1 & -1 & -1 & 0
\end{array}
$$

Note that Majorana mass terms for vanishing $U(1)_{R}$ charge combinations of $\nu_{R}$ 's are forbidden by the unbroken $Z_{3}^{\ell}$ symmetry. Also, for $Z_{3}^{\ell}$ to be anomaly free, three neutrinos must have $Z_{3}^{\ell}$ charge -1 while the other is neutral. The neutral one, $\nu_{R}^{4}$, can obtain a Majorana mass after the $U(1)_{R}$ breaking.

There are other possible $U(1)_{R}$ charge assignments, including chiral ones. In fact, as we show in Appendix B, there are no integer solutions to both requirements, Eqs. (20) and (21), with up to and including five righthanded neutrinos. With six neutrinos, one can find many solutions: $Q=(1,1,1,-4,-4,5),(1,1,5,-9,-9,11)$, etc. We expect the phenomenology of the model is more or less the same for such cases.

The Dirac neutrino masses are generated by the $\mathrm{U}(1)_{R}$ symmetry breaking in low energy. If we assume $\mathrm{U}(1)_{R}$ is

\footnotetext{
3 If we allow to introduce another discrete symmetry $Z_{2}, Q=$ $(+1,-1,0)$ is possible with assigning $Z_{2}$ odd for $\nu_{R}^{3}$. In this case, one of the three neutrinos remains massless.
} 
broken by the vev of a Higgs field $H_{R}$ with charge $-1 / n$, we can write down an operator similar to that in Eq. (12)

$$
\mathcal{L}^{R}=\lambda^{\alpha i}\left(\frac{H_{R}^{(*)}}{\Lambda}\right)^{n}\left(H L^{\alpha}\right) \nu_{R}^{i},
$$

where complex conjugation for $H_{R}$ is necessary for the generations with $Q_{R}=-1$. The $\mathrm{U}(1)_{R}$ breaking scale is the same as the discrete case and given in Eq. (13).

In the case of the charge assignment $Q=$ $(+1,-1,+1,-1)$, one of the right-handed neutrinos with vanishing $Z_{3}^{\ell}$ charge acquires a Majorana mass through the operator $\left(\nu_{R}^{4}\right)^{2} H_{R}^{2 n} / \Lambda^{2 n-1}$. In the chiral case with $Q=(1,1,1,-4,-4,5)$, one neutrino with charge +5 and one of the two neutrinos with charge -4 couple to $H_{R}^{n}$ to become a Dirac neutrino with mass of order $\left\langle H_{R}\right\rangle^{n} / \Lambda^{n-1}$. The other right-handed neutrino with charge -4 may have small Majorana masses of the order of $\left\langle H_{R}\right\rangle^{8 n} / \Lambda^{8 n-1}$.

After $U(1)_{R}$ symmetry breaking, the gauge field $A_{\mu}^{R}$ acquires a mass $m_{A} \sim q_{R} g_{R}\left\langle H_{R}\right\rangle$, where $q_{R}$ is the $U(1)_{R}$ charge of $H_{R}$. From Eq. (13), we expect $\left\langle H_{R}\right\rangle$ to be at least of order $1 \mathrm{eV}$. Therefore $m_{A} \gtrsim 10 \mathrm{eV}$ (assuming $\mathcal{O}(1)$ coupling constants), and hence $A_{\mu}^{R}$ does not mediate a long range force.

\section{Kinetic Mixing}

The neutrino sector and the electroweak sector are not completely decoupled. Kinetic mixing between $\mathrm{U}(1)_{R}$ and $\mathrm{U}(1)_{Y}$ arises at the renormalizable level, resulting in new interactions between $A_{\mu}^{R}$ and charged fermions of the SM. We parameterize the effective size of the mixing by $\varepsilon$ that we will treat as a perturbation, i.e., $\varepsilon \ll 1$.

Below the electroweak breaking scale, the gauge kinetic terms for the unbroken gauge symmetries are

$$
\mathcal{L}^{A}=-\frac{1}{4}\left(F_{\mu \nu} F^{\mu \nu}+F_{\mu \nu}^{R} F^{R \mu \nu}-2 \varepsilon F_{\mu \nu} F^{R \mu \nu}\right),
$$

where $F_{\mu \nu}^{(R)} \equiv \partial_{\mu} A_{\nu}^{(R)}-\partial_{\nu} A_{\mu}^{(R)}$. We consider the following redefinition of the fields ${ }^{4}$

$$
A_{\mu} \rightarrow A_{\mu}+\varepsilon A_{\mu}^{R} ; A_{\mu}^{R} \rightarrow A_{\mu}^{R}
$$

resulting in

$$
\mathcal{L}^{A}=-\frac{1}{4}\left(F_{\mu \nu} F^{\mu \nu}+F_{\mu \nu}^{R} F^{R \mu \nu}\right)+\mathcal{O}\left(\varepsilon^{2}\right) .
$$

With the gauge kinetic terms canonical, the charged fermions now couple to $A_{\mu}^{R}$ via

$$
Q_{f} \bar{\psi} \not A \psi \rightarrow Q_{f} \bar{\psi} \not A \psi+\varepsilon Q_{f} \bar{\psi} A_{R} \psi
$$

\footnotetext{
4 We note that $U(1)_{R}$ will be spontaneously broken, resulting in a massive vector boson. This redefinition is the only one that keeps the mass term for $A_{\mu}^{R}$ diagonal, and hence is the canonical choice.
}

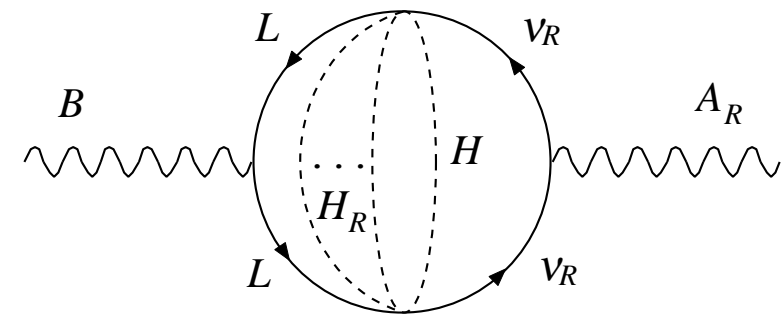

FIG. 1: $(2+n)$-loop diagram that radiatively induces kinetic mixing between $U(1)_{Y}$ and $U(1)_{R}$.

Hence, $A_{\mu}$ is identified as the usual photon, however, fermions of electric charge $Q_{f}$ have picked up a charge $Q_{R}=\varepsilon Q_{f}$ under $U(1)_{R}$.

What is the size of the kinetic mixing $\varepsilon$ ? Above the electroweak scale we can write

$$
\left(\varepsilon_{0}+\varepsilon_{2} \frac{H^{\dagger} H}{\Lambda^{2}}+\ldots\right) F_{\mu \nu}^{Y} F^{\mu \nu R}
$$

where $\varepsilon_{i}$ correspond to the coefficients of the $i+4$ dimensional operators. The effective value evaluated at the cutoff scale, $\varepsilon(\Lambda) \equiv \varepsilon_{0}+\varepsilon_{2} v^{2} / \Lambda^{2}+\ldots$, renormalizes to a low energy value $\varepsilon(\mu)$ through radiative corrections that appear at $(2+n)$-loops, see Fig. 1. We estimate the loop-induced kinetic mixing between $B$ and $A_{R}$ to be

$$
\varepsilon_{\text {rad }} \sim \frac{g_{Y} g_{R}}{\left(16 \pi^{2}\right)^{2+n}}
$$

where $g_{Y}=e / \cos \theta_{W}$ is the $U(1)_{Y}$ coupling and $g_{R}$ the $U(1)_{R}$ coupling. (At the electroweak scale this trivially matches to the kinetic mixing between the photon and $A_{R}, \varepsilon \sim\left(16 \pi^{2}\right)^{-2-n} e g_{R}$. $)$ Hence, it is technically natural to assume that the kinetic mixing at the cutoff scale is of the order of $\varepsilon(\Lambda) \sim\left(16 \pi^{2}\right)^{-2-n} g_{Y} g_{R}$. An appropriate UV completion could justify this assumption, for example by embedding $U(1)_{R}$ into an $S U(2)_{R}$ that is broken down to $U(1)_{R}$ at a low scale $\mu \ll \Lambda$, but we will not pursue this further. Instead, we will simply assume the cutoff scale value is zero and that the leading order contribution to the kinetic mixing arises only from the loop contributions. We now proceed to examine phenomenological constraints on the parameters of the model.

\section{Phenomenological Constraints}

We now consider several experimental bounds on the size of the kinetic mixing $\varepsilon$.

\section{Precision data}

Precision data on $g-2$ of the electron agree with the SM to better than 1 part in $10^{12}$. For $m_{A} \ll m_{e}$, this suggests that the one-loop contribution with $A_{R}$ in place 
of a photon is $\varepsilon^{2} \alpha / \pi$, and therefore $\varepsilon \lesssim 10^{-4}$ which is satisfied for any $n$. The case with $m_{A} \gg m_{e}$ is less constrained because of further suppression of $m_{e}^{2} / m_{A}^{2}$.

\section{Cosmic strings}

The spontaneous breaking of the $\mathrm{U}(1)_{R}$ gauge symmetry leads to formation of cosmic strings. The existence of the cosmic string causes discontinuities in the temperature of the CMB. The bound of $\Delta T / T \lesssim 10^{-5}$ puts a constraint on the breaking scale: $\left\langle H_{R}\right\rangle \lesssim 10^{-2.5} M_{P}$. By comparing this with Eq. (13), one finds it does not give a useful bound as long as we are interested in low-cutoff theories.

\section{3. $B B N$}

The number of relativistic degrees of freedom during BBN is constrained by the effective number of neutrino species $\Delta N_{\nu} \lesssim 1$ as we discussed with in the discrete Dirac neutrino case, Sec. IVA. All of the constraints that we found arising from the Yukawa interaction also apply to the continuous $\mathrm{U}(1)_{R}$ symmetry case.

Here we discuss additional constraints from the new gauge interaction. Since the QCD phase transition is sufficient to dilute any pre-existing abundance of $R$-sector particles, we need only consider processes that are in equilibrium below the temperature of the $\mathrm{QCD}$ phase transition. To delineate the restrictions on the parameter space, each production process can then be further divided into three cases depending on the mass of the gauge boson.

One important process is $e^{+} e^{-} \rightarrow \nu_{R} \bar{\nu}_{R}$ through $A_{R^{-}}$ exchange that occurs at a suppressed level proportional to the amount of kinetic mixing. We require that this process is not in equilibrium at whatever temperature (between $\mathrm{BBN}$ to the QCD phase transition) yields the maximum interaction rate. This gives the following constraints that depend on the gauge boson mass,

$$
\begin{aligned}
g_{R}^{2} \alpha \varepsilon^{2} T & \lesssim \frac{T^{2}}{M_{P}} \quad\left(m_{A}<1 \mathrm{MeV} ; T=1 \mathrm{MeV}\right) \\
\frac{g_{R}^{2} \alpha \varepsilon^{2} T^{5}}{m_{A}^{4}} & \lesssim \frac{T^{2}}{M_{P}} \quad\left(1 \mathrm{MeV}<m_{A}<200 \mathrm{MeV} ; T=m_{A}\right) \\
\frac{g_{R}^{2} \alpha \varepsilon^{2} T^{5}}{m_{A}^{4}} & \lesssim \frac{T^{2}}{M_{P}} \quad\left(m_{A}>200 \mathrm{MeV} ; T=200 \mathrm{MeV}\right)
\end{aligned}
$$

The resulting constraints are shown in Figs. 2 月.

The second process is the production of $A_{R}$ through for example $e^{+} e^{-} \rightarrow \gamma A_{R}$. Again, requiring that this process is not in equilibrium at the temperature that yields the maximum interaction rate yields the following constraints that depend on the gauge boson mass,

$4 \pi \alpha^{2} \varepsilon^{2} T \lesssim T^{2} / M_{P} \quad\left(m_{A}<1 \mathrm{MeV} ; T=1 \mathrm{MeV}\right)$

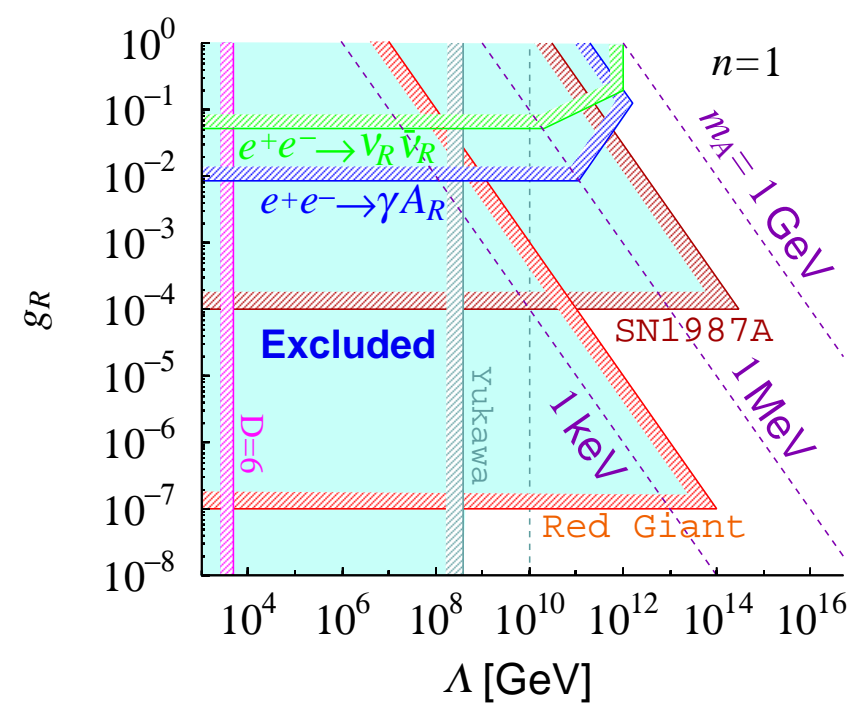

FIG. 2: The astrophysical constraints for the case $n=1$ are shown in the $\left(\Lambda, g_{R}\right)$ plane. The shaded region on the left-hand side is excluded. The constraints come from $e^{+} e^{-} \rightarrow \nu_{R} \bar{\nu}_{R}$ [Eqs. 29)]; $e^{+} e^{-} \rightarrow \gamma A_{R}$ [Eqs. (30)]; the $D=6$ operator Eq. (14); the Yukawa mediated processes $\nu_{L} \nu_{R} \rightarrow H_{R} H_{R}^{*} H_{R}^{*}$ or $\nu_{L} H_{R} \rightarrow \bar{\nu}_{R} H_{R} H_{R}^{*}$; overcooling red giants [Eq. (32)]; and overcooling SN1987A [Eq. (34)]. The diagonal dashed lines show contours of constant $m_{A}$. In the case of a discrete symmetry, all constraints except for Yukawa and $D=6$ disappear. If we assume the $R$-sector particles are in thermal equilibrium with the standard model particles before the QCD phase transition, the Yukawa constraint moves to the dotted line.

$$
\begin{aligned}
4 \pi \alpha^{2} \varepsilon^{2} T \lesssim T^{2} / M_{P} & \left(1<m_{A}<200 \mathrm{MeV} ; T=m_{A}\right) \\
\text { (no constraint) } & \left(m_{A}>200 \mathrm{MeV}\right)
\end{aligned}
$$

The resulting constraints are again shown in Figs. 2⿴囗大

\section{Red Giant and Supernova Cooling}

Astrophysical bounds from excessive energy loss through the emission of light, extremely weakly interacting particles from stars and supernovae are potentially much more severe if kinematically allowed. This is well known to put significant constraints on axions, and here we will use these bounds to constrain the emission rate of $A_{R}$.

Consider the emission of a light axion $a$, coupled to a fermion $\psi$ with strength $g_{\psi}$

$$
\mathcal{L}^{a}=-i g_{\psi} \bar{\psi} \gamma_{5} \psi a .
$$

Here, $g_{\psi}=m_{\psi} / f_{a}$, where $m_{\psi}$ is the fermion mass and $f_{a}$ is the effective Peccei-Quinn scale. Using astrophysical data on red giants, one finds, for $\psi=e, g_{e} \lesssim 2.5 \times 10^{-13}$, corresponding to roughly $f_{a} \gtrsim 10^{9} \mathrm{GeV}[18,19]$.

Axion emission proceeds through the pseudoscalar interaction, Eq. (31), that vanishes in the non-relativistic 


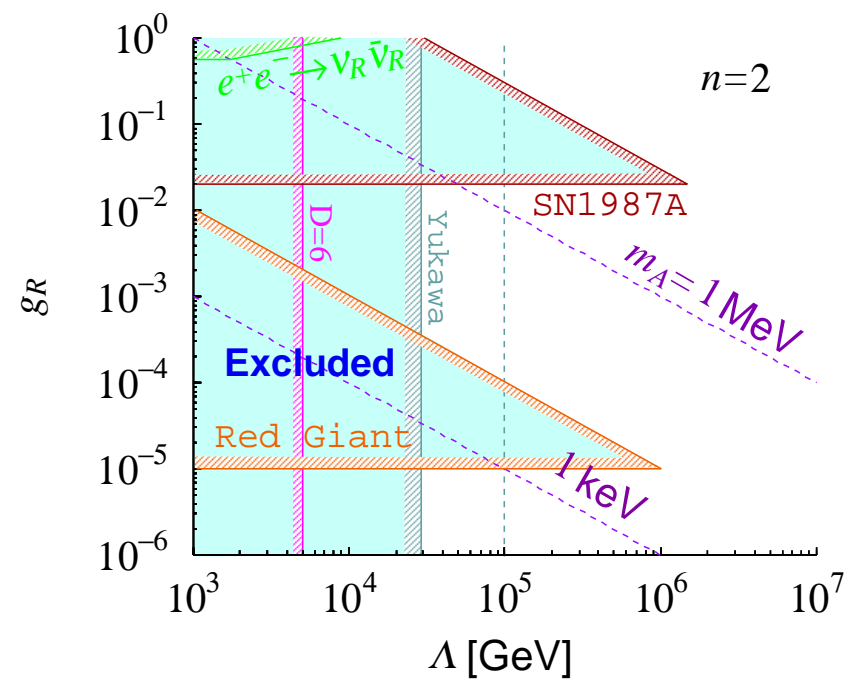

FIG. 3: Same as Fig. 2 but for $n=2$ and the scales are quite different.

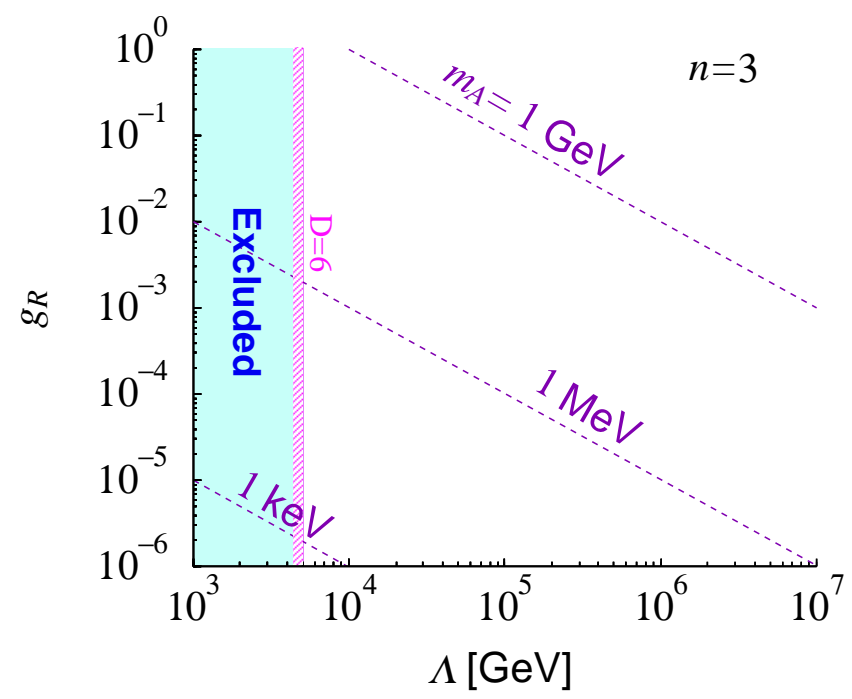

FIG. 4: Same as Fig. 3 but for $n=3$.

limit. Emission of $A_{R}$, however, proceeds through the usual vector interaction without any non-relativistic suppression. This means that the amplitude for the emission of $A_{\mu}^{R}$ off an electron is expected to be enhanced by an amount $\sim m_{e} / T_{\text {giant }} \sim 50$ with respect to axion emission, where $T_{\text {giant }} \sim 10 \mathrm{keV}$ is the core temperature of the red giant. Using $\varepsilon \sim 50 g_{e}$, we obtain the stringent upper limit $\varepsilon \lesssim 10^{-14}$ when $m_{A} \lesssim T_{\text {giant }}$. It is straightforward to translate the constraints on the parameters $\varepsilon$ and $m_{A}$ into constraints on $g_{R}$ and $\Lambda$. We obtain the following exclusion regions:

$$
\begin{array}{llll}
n=1: & g_{R} \gtrsim 10^{-7} & \text { or } & g_{R} \Lambda \lesssim 10^{7} \mathrm{GeV} \\
n=2: & g_{R} \gtrsim 10^{-5} & \text { or } & g_{R} \Lambda \lesssim 10 \mathrm{GeV}
\end{array}
$$

for the two cases. The excluded region for the cases $n=$ 1,2 are shown as the lower triangular regions in Figs. 2, 3 .
Given the range of scales shown in Fig. 4 , there is no constraint for $n \geq 3$.

Are $A_{R}$ 's ever strongly enough coupled to matter to be trapped in red giants? Assuming the scattering of $A_{R}$ is dominated by Compton scattering, the cross section can be estimated as $\sigma \sim \varepsilon^{2} \alpha^{2} m_{e}^{-2} \sim \varepsilon^{2} 10^{-25} \mathrm{~cm}^{2}$. Using $n_{e} \sim 10^{30} \mathrm{~cm}^{-3}$ as the average number density of electrons in the core, we obtain $d \sim\left(n_{e} \sigma\right)^{-1} \sim \varepsilon^{-2} 10^{-5} \mathrm{~cm}$. This can be written as

$$
d \sim \frac{10^{5+4 n} \mathrm{~cm}}{g_{R}^{2}}
$$

This distance is larger than the core radius of a red giant, $R_{\text {core }} \sim 10^{9} \mathrm{~cm}$, regardless the dimension of the operator or other parameters so long as $g_{R} \lesssim 1$.

We note that assuming $g_{R} \gtrsim 1$ is not a viable solution either. In this case, we have $m_{A} \sim g_{R}\left\langle H_{R}\right\rangle \gtrsim$ $100 \mathrm{MeV}$, given our previous bounds. However, in the examples that we have considered, there is always a $\nu_{R}$ with small $(\ll 1 \mathrm{eV})$ Majorana mass in the spectrum. The decay rate of $A_{R}$ is then bounded from below by $g_{R}^{2} m_{A} \gtrsim 100 \mathrm{MeV} \sim 10^{22} \mathrm{~s}^{-1}$. Thus, $A_{R}$ quickly decays into $\nu_{R} \bar{\nu}_{R}$ which, in turn, escape the star. Hence, $A_{R}$ particles cannot be trapped in a red giant in any regime of parameters.

The observation of neutrinos from SN1987A can also be used to place bounds on the emission of axions and $A_{R}$. Axions are emitted via bremsstrahlung off of nucleons in the nucleon scattering process $N N \rightarrow N N a$ via pion exchange [20]. As a result, the axion-nucleon coupling range $3 \times 10^{-10}<g_{N}<3 \times 10^{-7}$ is ruled out [16]. Values larger than the upper limit are allowed for axions due to the possibility of trapping inside the supernova.

Translating this bound on axions into a bound on $\varepsilon$, there is again a relative enhancement of the $A_{R}$ coupling to nucleons since it does not have a non-relativistic suppression compared with axions. The coupling enhancement is roughly $m_{N} / T_{\mathrm{SN} 1987 \mathrm{~A}} \sim 30$, where $T_{\mathrm{SN} 1987 \mathrm{~A}} \sim$ $30 \mathrm{MeV}$, suggesting that the range $10^{-11}<\varepsilon<10^{-8}$ is excluded so long as $m_{A_{R}} \lesssim T_{\mathrm{SN} 1987 \mathrm{~A}}$. This implies $g_{R} \gtrsim 0.1$ for $n \geq 1$ may be allowed for trapped $A_{R}$ 's. However, this is not the case. In a similar fashion as in the case of red giants, we get an upper bound on the lifetime of $A_{R}$ of order $10^{-20} \mathrm{~s}$. This is much shorter than the typical cooling timescales for a supernova, which is of order seconds. The fast decay $A_{R} \rightarrow \nu_{R} \bar{\nu}_{R}$ then results in overcooling of the supernova and trapping is again irrelevant here. We thus obtain the following exclusion bounds:

$$
\begin{array}{llll}
n=1: & g_{R}>10^{-4} & \text { unless } & g_{R} \Lambda>10^{11} \mathrm{GeV} \\
n=2: & g_{R}>0.02 & \text { unless } & g_{R} \Lambda>50 \mathrm{TeV}
\end{array}
$$

The excluded region of parameters for $n=1,2$ is shown in Figs. 2 and 3, respectively. There is no constraint for $n \geq 3$. 


\section{HIERARCHY}

Our models contain a (smaller) hierarchy problem of their own: the hierarchy between the gauge symmetry breaking scales and the cutoff scale. In Majorana case, we considered symmetry breaking in the infrared, in the range $2-400 \mathrm{keV}$. It is possible that this scale could be stabilized by supersymmetry. In the effective theory below the electroweak scale, the scalar fields $\chi$ acquire a 1-loop quadratically divergent contribution proportional to $g^{2}$. If this were the only contribution to the scalar (mass) ${ }^{2}$, the hierarchy would be stabilized. However, there are cutoff suppressed contributions such as $Z^{\dagger} Z \chi^{\dagger} \chi / \Lambda^{2}$, where $Z$ is a hidden sector field with an $F$ term, that are not forbidden by symmetries. One possibility is to remove these operators through sequestering, thereby allowing anomaly mediation to dominate 21. One might be concerned about the quadratic divergence of the scalar masses below the supersymmetry breaking scale. This arises at two- or three-loops, giving a contribution at most of order $m_{\chi}^{2} \sim m_{\mathrm{SUSY}}^{6} /\left(\left(16 \pi^{2}\right)^{3} \Lambda^{4}\right)$. But, it is easy to see that so long as the scale of the soft breaking $m_{\mathrm{SUSY}} \lesssim 4 \pi v$, this higher order contribution is subdominant to the already safe one-loop quadratically divergent contribution below the electroweak breaking scale discussed above.

Another way to stabilize this hierarchy is to use technicolor. Consider the Dirac case for purposes of illustration. Suppose there is a new $S U(3)$ gauge group with dynamical scale $\Lambda_{R}$, that couples to two triplets $q_{i}\left(\mathbf{3}_{0}\right)$ with $i=1,2$ and two anti-triplets $\bar{q}_{+}\left(\overline{\mathbf{3}}_{+1}\right)$ and $\bar{q}_{-}\left(\overline{\mathbf{3}}_{-1}\right)$. The subscripts here refer to the $U(1)_{R}$ charges. Note that the gauge invariance completely forbids mass terms for techniquarks. The theory is identical to the two-flavor QCD and is known to cause a condensate $\left\langle\bar{q}_{+} q_{1}\right\rangle=\left\langle\bar{q}_{-} q_{2}\right\rangle \simeq \Lambda_{R}^{3} \neq 0$ that breaks $U(1)_{R}$. This model corresponds to $n=3$ case in Eq. (22) with $H_{R}^{3}$ replaced by the fermion bilinear

$$
\mathcal{L}^{R}=\lambda^{\alpha i} \frac{\left(H L^{\alpha}\right)\left[\left(\bar{q}-q_{2}\right) \nu_{R}^{i}\right]}{\Lambda^{3}} .
$$

While this mechanism stabilizes $\Lambda_{R}$ against $\Lambda$, and naturally leads to the small Dirac neutrino masses, the $\mathrm{U}(1)$ symmetry breaking scale is much larger, $\Lambda_{R} \sim$ $\left(m_{\nu} / v\right)^{1 / 3} \Lambda \gtrsim 10^{-4} \Lambda$. Choosing a cutoff $\Lambda \gtrsim 100 \mathrm{TeV}$ (to avoid FCNC problems in the SM) implies $\Lambda_{R} \gtrsim 10$ $\mathrm{GeV}$.

We note that lowering the cutoff scale of the Standard Model may also be constrained by too-large flavor changing neutral current processes from cutoff scale suppressed flavor-violating operators. The Majorana case and the $n \leq 2$ Dirac cases are roughly safe from these constraints, since we found the cutoff scale must be larger than tens of $\mathrm{TeV}$. (This is curiously reminiscent of the smallest cutoff scale that was found by using split fermions as a solution to global symmetry breaking [1.) For $n \geq 3$ in the Dirac case, the flavor problem becomes more acute as the cutoff scale is taken to the lowest possible value, of order $5 \mathrm{TeV}$.

\section{CONCLUSION}

The hierarchy problem may be solved by lowering the cutoff scale of the SM. However, this leads to unacceptably large violations of baryon and lepton numbers. We have shown there are simple discrete gauge symmetries that can be imposed on the SM to protect against rapid proton decay and too-large Majorana neutrino mass. We then constructed models that generate small neutrino mass through spontaneous (discrete or continuous) gauge symmetry breaking at small scales.

In our first model, Majorana neutrino mass were generated once $Z_{3}^{\ell}$ is broken. This model is restricted by the experimental constraints from the non-observation of $0 \nu \beta \beta \chi$, which requires $\Lambda>30 \mathrm{TeV}$, and the nonobservation of modifications to $\Delta T / T$ in the CMB from a (non-frustrated) domain wall network, which requires $\Lambda \lesssim 400 \mathrm{TeV}$. Generating neutrino masses near the observed size implies the scale for $Z_{3}^{\ell}$ breaking is of order 2-400 keV. Values near the upper end of this range yield domain walls in the universe that modify the CMB at observable levels. If the domain wall network were frustrated, such as if $Z_{3}^{\ell}$ came from a larger discrete symmetry, the domain walls could provide the dark energy of the universe with $w=-2 / 3$.

Dirac-neutrino masses arise when $Z_{3}^{\ell}$ is exact and righthanded neutrinos are added to the model. We proposed a new discrete and $U(1)_{R}$ symmetry that acts only on right-handed neutrinos. Dirac neutrino masses of the observed size are generated once the symmetry is broken. Limiting the light degrees of freedom during $\mathrm{BBN}$ gives the strongest constraint on the models. We found that the models with a discrete symmetry are disfavored by the domain wall constraint. Models with a U(1), however, are viable with $\Lambda \gtrsim 5-30 \mathrm{TeV}$ when the dimension of the operator that generates the Dirac neutrino masses is more than six $(n \geq 2)$. More precise constraints are shown in the figures. The new gauge boson mass is $m_{A} \simeq g_{R} 100 \mathrm{MeV}$ for $n=2$ and higher for larger $n$. If the gauge coupling is small, a very light gauge boson is possible.

\section{APPENDIX A: ALTERNATIVE DISCRETE LEPTON NUMBER}

Note that flavor-blindness in the lepton sector can be relaxed with interesting consequences for allowed FCNC operators. For example, the assignment

$$
\begin{array}{c|cccccc} 
& L_{e} & e_{e}^{c} & L_{\mu} & e_{\mu}^{c} & L_{\tau} & e_{\tau}^{c} \\
\hline Z_{5}^{\ell} & -2 & 2 & 1 & -1 & 1 & -1
\end{array}
$$

forbids not only Majorana masses for neutrinos, but also the flavor-changing lepton decay $\mu \rightarrow e \gamma$. Majorana neu- 
trino masses with arbitrary flavor mixing can be generated with two fields $\chi_{1,2}$ with charges $-1,-2$ that acquire vevs and spontaneously break the $Z_{5}^{\ell}$ symmetry. The process $\mu \rightarrow e \gamma$ also reappears, but now further suppressed by $\left\langle\chi_{i}\right\rangle / \Lambda$. While we find this an amusing option, we adopt the flavor-blind $Z_{3}^{\ell}$ in the body of the paper.

\section{APPENDIX B: $U(1)$ CHARGES}

In this appendix, we show that there are no anomalyfree charge assignments with fewer than six neutrino species that are (1) chiral, and (2) two of the charges are the same.

It is easy to show that there are no chiral charge assignments up to four neutrinos. Without a loss of generality, we can always choose one of the charges to be +1 . With four neutrinos, the charges are $Q=(1, \alpha, \beta, \gamma)$. The anomaly freedom requires

$$
\begin{aligned}
1+\alpha+\beta+\gamma & =0 \\
1+\alpha^{3}+\beta^{3}+\gamma^{3} & =0 .
\end{aligned}
$$

Solving for $\gamma$ in the first equation and substituting into the second, we find

$$
-3(1+\alpha)(1+\beta)(\alpha+\beta)=0 .
$$

All solutions to this equation give vector-like charge assignments: For $\alpha=-1$, we find $Q=(1,-1, \beta,-\beta)$. For $\beta=-1$, we find $Q=(1, \alpha,-1,-\alpha)$. For $\alpha=-\beta$, we find $Q=(1, \alpha,-\alpha,-1)$.

With five neutrino species, one can find non-trivial chiral charge assignments. There are integer solutions, such as $Q=(1,5,-7,-8,9)$ or $(2,4,-7,-9,10)$. However, with only one Higgs that breaks the $U(1)_{R}$ gauge symmetry, we need at least two neutrino species to have the same charge so that both of them can acquire masses. Without a loss of generality, we can normalize the charges such that two species have charge unity, $Q=(1,1, \alpha, \beta, \gamma)$. Then the anomaly freedom requires

$$
2+\alpha+\beta+\gamma=0
$$

$$
2+\alpha^{3}+\beta^{3}+\gamma^{3}=0
$$

Substituting the solution for $\alpha$ from the first equation to the second, we find

$$
\begin{aligned}
& \alpha=-\frac{\gamma^{2}+4 \gamma+4 \pm \sqrt{\gamma\left(\gamma^{3}-8 \gamma-8\right)}}{4+2 \gamma}, \\
& \beta=-\frac{\gamma^{2}+4 \gamma+4 \mp \sqrt{\gamma\left(\gamma^{3}-8 \gamma-8\right)}}{4+2 \gamma} .
\end{aligned}
$$

Therefore, for any choice of $\gamma$, we can always find appropriate $\alpha$ and $\beta$.

However, there are no integer solutions. The integer solutions would require that $\alpha, \beta$, and $\gamma$ are all rational numbers. We can always write $\gamma=p / q$, where $p$ and $q$ are relatively prime. In order for $\alpha$ and $\beta$ to be also rational, the argument of the square root

$$
\gamma\left(\gamma^{3}-8 \gamma-8\right)=\frac{p(p+2 q)\left(p^{2}-2 p q-4 q^{2}\right)}{q^{4}},
$$

must be a complete square. Therefore, the numerator on the r.h.s. must be a complete square of an integer. Because there is a factor of $p$ already, other two factors must contain a factor of $p$. However, both $p+2 q$ and $p^{2}-2 p q-4 q^{2}$ are relatively prime with $p$ because $q$ is relatively prime with $p$. Q.E.D.

\section{ACKNOWLEDGMENTS}

H.D. was supported in part by the P.A.M. Dirac Fellowship, awarded by the Department of Physics at the University of Wisconsin-Madison. This work was supported by the IAS, funds for Natural Sciences, as well as in part by the DOE under contracts DEFG02-95ER40896, DE-FG02-90ER40542 and DE-AC0376SF00098, and in part by NSF grants PHY-0098840 and PHY-0070928.
[1] H. Davoudiasl, R. Kitano, T. Li and H. Murayama, arXiv:hep-ph/0405097.

[2] N. Arkani-Hamed, S. Dimopoulos and G. R. Dvali, Phys. Lett. B 429, 263 (1998) [arXiv:hep-ph/9803315].

[3] L. Randall and R. Sundrum, Phys. Rev. Lett. 83, 3370 (1999) [arXiv:hep-ph/9905221].

[4] N. Arkani-Hamed and M. Schmaltz, Phys. Rev. D 61, 033005 (2000) [arXiv:hep-ph/9903417].

[5] Y. Grossman and M. Neubert, Phys. Lett. B 474, 361 (2000) [arXiv:hep-ph/9912408].

[6] L. E. Ibanez and G. G. Ross, Phys. Lett. B 260, 291 (1991); L. E. Ibanez and G. G. Ross, Nucl. Phys. B 368, 3 (1992).
[7] K. S. Babu, I. Gogoladze and K. Wang, Phys. Lett. B 570, 32 (2003) [arXiv:hep-ph/0306003].

[8] Z. Chacko, L. J. Hall, T. Okui and S. J. Oliver, arXiv:hep$\mathrm{ph} / 0312267$.

[9] Z. Chacko, L. J. Hall, S. J. Oliver and M. Perelstein, arXiv:hep-ph/0405067.

[10] T. Banks and M. Dine, Phys. Rev. D 45, 1424 (1992) [arXiv:hep-th/9109045].

[11] H. M. Georgi, S. L. Glashow and S. Nussinov, Nucl. Phys. B 193, 297 (1981).

[12] T. Bernatowicz, J. Brannon, R. Brazzle, R. Cowsik, C. Hohenberg and F. Podosek, Phys. Rev. Lett. 69, 2341 (1992). 
[13] A. Friedland, H. Murayama and M. Perelstein, Phys. Rev. D 67, 043519 (2003) [arXiv:astro-ph/0205520].

[14] L. Conversi, A. Melchiorri, L. Mersini and J. Silk, Astropart. Phys. 21, 443 (2004) [arXiv:astro-ph/0402529].

[15] A. Pierce and H. Murayama, Phys. Lett. B 581, 218 (2004) [arXiv:hep-ph/0302131]; P. Crotty, J. Lesgourgues and S. Pastor, Phys. Rev. D 67, 123005 (2003) [arXiv:astro-ph/0302337]; V. Barger, J. P. Kneller, H. S. Lee, D. Marfatia and G. Steigman, Phys. Lett. B 566, 8 (2003) [arXiv:hep-ph/0305075];

[16] S. Eidelman et al. [Particle Data Group Collaboration], Phys. Lett. B 592, 1 (2004).

[17] V. Barger, J. P. Kneller, P. Langacker, D. Marfatia and G. Steigman, Phys. Lett. B 569, 123 (2003) [arXiv:hep- $\mathrm{ph} / 0306061]$.

[18] G. Raffelt and A. Weiss, Phys. Rev. D 51, 1495 (1995) [arXiv:hep-ph/9410205].

[19] G. G. Raffelt, Ann. Rev. Nucl. Part. Sci. 49, 163 (1999) [arXiv:hep-ph/9903472].

[20] N. Iwamoto, Phys. Rev. Lett. 53, 1198 (1984); R. P. Brinkmann and M. S. Turner, Phys. Rev. D 38, 2338 (1988); A. Burrows, M. T. Ressell and M. S. Turner, Phys. Rev. D 42, 3297 (1990).

[21] L. Randall and R. Sundrum, Nucl. Phys. B 557, 79 (1999) [arXiv:hep-th/9810155]; G. F. Giudice, M. A. Luty, H. Murayama and R. Rattazzi, JHEP 9812, 027 (1998) [arXiv:hep-ph/9810442]. 